\title{
SIMULATION TOOL FOR SCANNING X-RAY BEAMS IRRADIATOR
}

\author{
V. T.Lazurik, V. M. Lazurik, G. F.Popov, Yu.V.Rogov \\ Kharkov National University, P.O. Box 60, 61052 Kharkov, Ukraine
}

\begin{abstract}
X-ray (bremsstrahlung) produced by high-power electron accelerators are intensively used in different radiation technologies. An electron accelerator, a scanner, an X-ray converter with cooling system, a conveyor line or a turntable chamber, an irradiated product and a package are the major components of the radiation technological lines (RTL) for X-ray irradiator. The detailed physical and geometrical models of the X-ray irradiator were realized in the form of new mathematical software of the "XR-Soft". The program XR-Soft was designed specially for simulation of industrial radiation processes and calculation of the absorbed dose and temperature distribution within products irradiated by scanning X-ray beams on industrial RTL that is based on the pulsed or continuous type of electron accelerators in the energy range from 0.1 to $50 \mathrm{MeV}$. The motivation for the development of the program XR-Soft is the creation of accurate and easily accessible tool for prediction of the absorbed dose distribution within irradiated materials, for optimization of the irradiation regimes of radiation facility, and for reducing of experimental routine dosimetric measurements in X-ray processing.
\end{abstract}

\section{INTRODUCTION}

The conception of the Radiation-Technological Office (RT-Office) was developed by authors. RTOffice realize computer technologies at all stages of works execution on the RTL for electron beam (EB), $\mathrm{X}$-ray and $\gamma$-ray processing. It is beginning with the expertise of the consumer order for scientifically-justified definition of the requirements and conditions for realization of an irradiating and ending with a provision of argued report on the fulfilled work.

The RT-Office is the common program shell which provides flexible intellectual interaction between specialized modules and databases for optimal planning of the process of an irradiating and control of its realization. Simulation and calculation modules of the RT-Office are the basis for construction of the specialized software for EB, X-ray and $\gamma$-ray processing $[1,2,3]$.

The modules structure, geometrical and physical models of the X-ray irradiator for the program XR-soft that was constructed from the RT-Office modules and is intended for simulation of X-ray processing are considered in the paper more closely.

\section{XR-SOFT STRUCTURE}

Two geometrical models for X-ray irradiators that were used for simulation X-ray processing are shown in Fig.1 and Fig.2. The first X-ray irradiator includes an electron accelerator, a scanner of electron beam, an X-ray converter with cooling system, and an irradiated product with packaging on moving conveyor (Fig.1). The second one includes an irradiated product placed in turntable cylindrical chamber that is rotating in front of the X-ray beam (Fig.2).

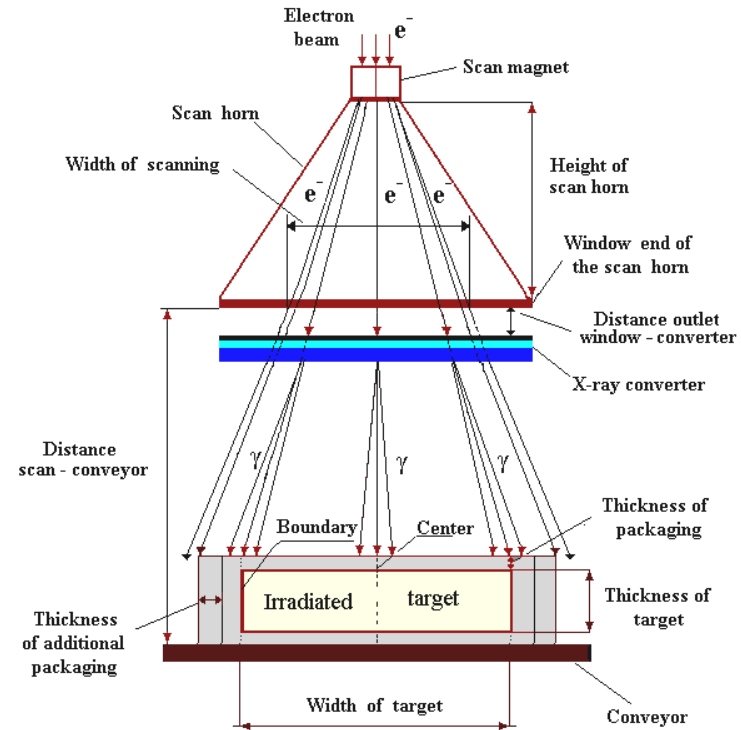

Figure 1. Scheme of radiation facility with X-ray converter for triangular-shaped scan horn and moving conveyor.

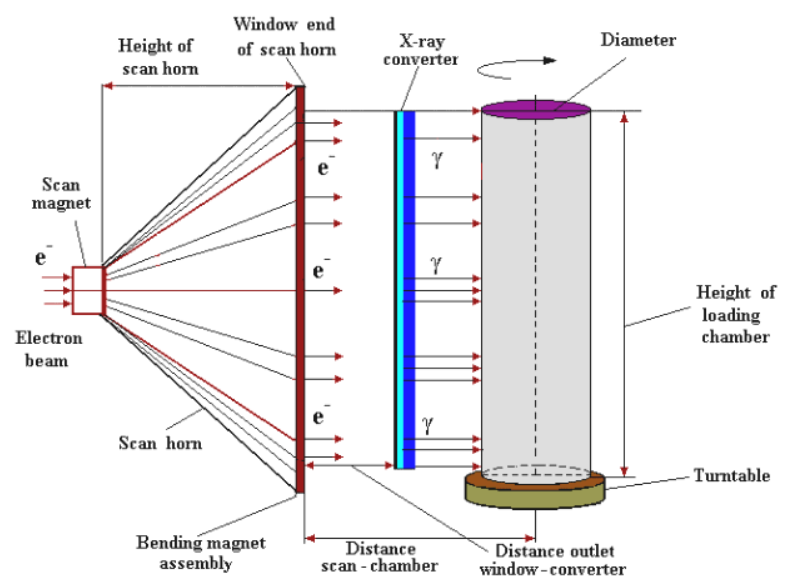

Figure 2. Scheme for X-ray irradiator with turntable loading chamber and non-diverging (parallel ray) EB.

The self-consistent physical models relatively each geometrical models of the X-ray irradiator were constructed on the base of the RT-Office simulation and calculation modules.

These modules are the following: 
- Monte Carlo simulation of dose distribution for electron beam into heterogeneous targets.

- Monte Carlo simulation of electron-photon shower in X-ray converter.

- Monte Carlo simulation of dose distribution into heterogeneous targets irradiated by X-ray beam on moving conveyor.

- Monte Carlo simulation of dose distribution for cylindrical turntable target irradiated by X-ray beam.

- Calorimetry. Calculation of spatial distribution of radiation-induced temperature and analytical estimations of integral characteristics of a heat transmission for process of cooling of the irradiated products in a thermostable environment.

- Comparison. Methods of mathematical physics for handling and comparative analysis of depth dose curves obtained by different calculation and experimental methods.

- Dosimetry. Specialized tool for loading and processing of experimental dosimetric data and their transmission to the Comparison module.

- RTL configuration. Loading and saving of the operational characteristics for RTL facility.

- Wizard for control and validation of input data for working regimes of RTL.

- Cognitive visualization of results for 2-dimensional (2-D) and 3-D view of dose distribution.

- The processing technologies database for equipment characteristics and objects used in radiation technologies.

Simulation of X-ray dose mapping in irradiated target materials was conducted by Monte Carlo method in 2-D model. The 2-D dose distribution in the target irradiated by $\mathrm{X}$-ray beam on moving conveyer is represented as function of two coordinates - of the target depth (axis $\mathrm{X}$ ) and the target width along scan direction (axis Y). The 2-D dose distribution into cylindrical turntable target is represented as function of the target radius (axis $\mathrm{X}$ ) and the target height (width) along scan direction (axis Y). Such conditions are realized in many practical cases for X-ray beam processing.

Features of realization of a physical model for X-ray processing are the following:

- The use of a forced method for process of producing $\mathrm{X}$-ray on each step of design of electron track in a construction of the X-ray converter.

- The automatic choice of self-consistent parameters is used for simulation of an electron - photon shower. The choice is based on determination a minimum machine time for obtaining given accuracy. These parameters are the following: cutoff energy for modeling of an electron track, threshold energy of catastrophic electron-electron collisions, cutoff energy for modeling of a photon track, threshold angle of grouping electron collisions for modeling of scattering.

- The use of both a simple estimation (collision method) and the special estimation (method of crossing area) for the dose calculation.
These features allow to reduce the running time of Monte Carlo simulation for receiving of the end results in about hundreds time. The program XR-Soft takes into account in detail a construction of the RTL and requirements to regimes of irradiation in each specific radiation-technological process.

\section{SIMULATION OF X-RAY DOSE MAPPING}

The processing rate and $\mathrm{X}$-ray absorbed dose distribution within of the irradiated materials depend on a lot of parameters of the radiation facility of RTL and characteristics of target material. Input data for the program XR-Soft are the following: Parameters of electron beam: average beam current, or pulse duration and repetition frequency in pulsed accelerators, electron spectrum, beam diameter and spatial distribution of the beam intensity. Parameters of scanning system: modes of operation, the triangular or non-diverging irradiation treatment field in target material; form of current in magnet of scanning system; repetition frequency of scanning; angular distribution of electron beam at the outlet of a scanning system; parameters of the exit window for electron beam. Parameters of the X-ray converter with cooling system: geometrical characteristics of the X-ray converter with cooling system, thickness of plates (layers) and cooling agent, materials composition, distance between exit window and X-ray converter. Parameters of conveyor line: speed and geometrical characteristics of the line. Parameters of irradiated product: geometrical characteristics of the irradiated product; elemental composition of the target; material and size of the covering for irradiated product. Regimes of target irradiation: one-, two-sided irradiation on moving conveyer, and irradiation of turning target.

For searching optimum solution, the program XR-Soft calculates and represents an X-ray energy spectrum, an absorbed dose distribution within irradiated products, a dose uniformity ratio, an optimum product thickness, an $\mathrm{X}$-ray utilization efficiency in the irradiated products, an electron beam power utilization, and other important characteristics for economic evaluation of X-ray processing.

X-ray dose mapping within compound (wood of aspen $+70 \%$ polymethylmethacrylate (PMMA)) with density $0.8 \mathrm{~g} / \mathrm{cm}^{3}$ for optimal target thickness at doublesided irradiation for saw-tooth and special forms of current in scan magnet are shown in Fig. 3 (a) and (b) respectively. X-ray beam was generated by scanning electron beam with electron energy $5 \mathrm{MeV}$ in a tantalum converter. The optimal converter construction includes the tantalum target plate with thickness $1.2 \mathrm{~mm}$, the cooling water channel $-3 \mathrm{~mm}$ and the aluminum backing plate $-5.0 \mathrm{~mm}$. The X-ray yield in the forward direction for $5 \mathrm{MeV}$ electron is $8.71 \%$. Regimes irradiation: electron beam energy - $5 \mathrm{MeV}$; beam current - $1 \mathrm{~mA}$; triangular scanning; width of target $-100 \mathrm{~cm}$; width of scanning $-100 \mathrm{~cm}$; conveyer speed $-1 \mathrm{~cm} / \mathrm{s}$. Target has not cover box. The optimal thickness for maximum X-ray 
power utilization is $38.5 \mathrm{~cm}$ relatively of dose distribution at the center of a target. X-ray beam power utilization in this case is $58 \%$.

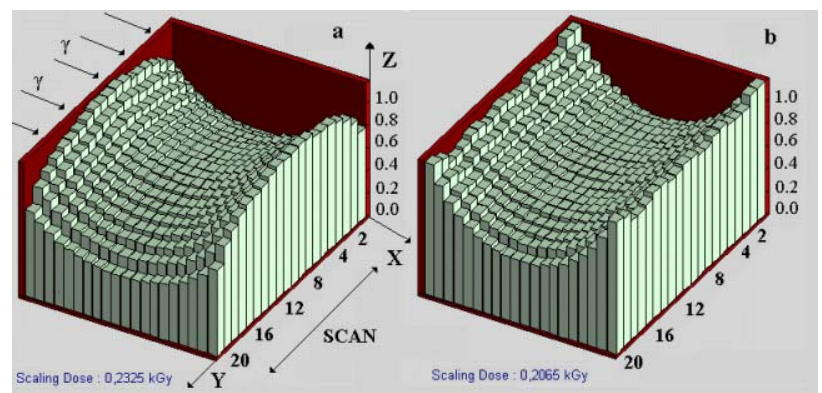

Figures 3 (a) and (b). X-ray dose mapping within compound for optimal target thickness and for the sawtooth form of current (a) and the special form of current (b) in scan magnet. Statistical deviations: $2.2 \%$ for center target and $2.5 \%$ for boundary target. (Running time is about 8 minutes on PC AMD-K7, $750 \mathrm{MHz}$ ).

For each product to be treated in the X-ray irradiation facility, there will usually be a minimum dose limit $\boldsymbol{D}_{\text {min-lim }}$ to obtain the desired effect and a maximum dose limit $\boldsymbol{D}_{\text {max-lim }}$ to avoid product degradation. As is seen from Fig. 3(a), the X-ray depth-dose distribution within compound has minimal value on the boundaries of a target along direction of the scanning X-ray beam and maximal value at plane that cross the target center. From standpoint of the dose limits, the minimum dose limit $\boldsymbol{D}_{\text {min-lim }}$ must be chosen as a minimum dose value $\boldsymbol{D}_{\text {min- }}$ bound on the boundaries of an irradiated target, the maximum dose limit $\boldsymbol{D}_{\boldsymbol{m a x} \text {-lim }}$ - as a maximum dose value $\boldsymbol{D}_{\text {max-center }}$ in the target center. In this case the dose uniformity ratio will be determined for all irradiated volume as $\mathrm{DUR}_{\mathrm{V}}=\boldsymbol{D}_{\text {max-center }} / \boldsymbol{D}_{\text {min-bound }}$.

For the center of a target the dose uniformity ratio DUR $=D_{\max } / D_{\min }$ is 1.51 . For the target boundary the DUR is 1.94. The value $D_{U} R_{V}$ is 2.91. Significant dose gradient in volume of irradiated target in direction of X-ray scanning can be decrease by the choice of the special shape of current in scan magnet $[2,6]$. By the use the program XR-Soft, the special shape of current in scan magnet which provide the maximum uniformity for $\mathrm{X}$-ray dose distribution in the irradiated compound was determined (see Fig. 3(b)). In this case X-ray beam power utilization is $53 \%$ and the value $\mathrm{DUR}_{\mathrm{V}}$ is 2.1 and also is greater than DUR in the target center.

Further decreasing of the target thickness at double sided X-ray treatment results in decreasing both the value $\mathrm{DUR}_{\mathrm{V}}$ and the $\mathrm{X}$-ray power utilization. For example, for compound thickness $\mathrm{h}=30 \mathrm{~cm}$ the $\mathrm{DUR}_{\mathrm{V}}$ is 2.0 for saw-tooth shape of current and the $D_{U} R_{V}$ is 1.6 for the special shape of current in scan magnet. The $\mathrm{X}$-ray power utilization is $48 \%$.

Most effectively X-ray processing can be realized by treatment of turning loading chamber with irradiated materials which is placed in front of scanning X-ray beams [7]. In this case at given value $\mathrm{DUR}_{\mathrm{V}}$ an optimal material thickness for X-ray processing will be greater than for double-sided irradiation. The program XR-Soft allows to simulate a dose mapping in turning cylinder chamber with materials irradiated by scanning X-ray beam. The direction of scanning X-ray beam is in parallel with a cylinder axis. For above compound and X-ray parameters, for the value $\mathrm{DUR}_{\mathrm{V}}=1.54$, the optimal thickness (diameter) for the treated material is $60 \mathrm{~cm}$.

The testing and verification of the results simulated by the program XR-Soft were carried out in compare with theoretical calculated data, with results obtained by the universal packages such as ITS, EGS4 and PENELOPE, and experimental data set in published works $[4,5]$. The comparison investigations indicated that the developed physical and mathematical models are reliable and correct, and the program XR-Soft is accurate, easily accessible for all users with a different level of background in an area of computer technologies.

\section{CONCLUSION}

X-ray dose mapping must be conducted with consideration of the dose non-uniformity in the target center as well as on the boundaries of an irradiated target along direction of the scanning X-ray beam. A non-uniformity equalization for X-ray dose distribution along a direction of scanning can be done by the choice of the special shape of current in scan magnet, with help of the special filters, or with special methods of irradiation [2]. As a minimum, the 2-D simulation model for X-ray dose mapping must be used for correct analysis of the optimum product thickness, X-ray power utilization, $\max / \mathrm{min}$ dose ratio, and $\mathrm{X}$-ray processing capacity. The program XR-Soft can be used as predictive tool for X-ray dose mapping, for determination of location $\boldsymbol{D}_{\min }$ and $\boldsymbol{D}_{\max }$ in target irradiated by scanning X-ray beams on RTL, and for optimization of regimes $\mathrm{X}$-ray irradiation to receive the maximum processing capacity with the minimum for dose uniformity ratio.

\section{REFERENCES}

[1] V.T. Lazurik, V.M. Lazurik, G.F.Popov, Yu.V. Rogov and S.A Korenev. Proc. of the PCaPAC, 2000, DESY, Hamburg, Germany, p.4. CD-Rom.

[2] V.T. Lazurik, V.M. Lazurik, G.F.Popov, Yu.V.Rogov Abstract book of the ICAP-2002 Conference, p.49, East Lansing, MI, USA. (Paper in press).

[3] V.T. Lazurik, V.M. Lazurik, G.F.Popov, Yu.V. Rogov Abstract Book of the PCaPAC 2002, P.32, Frascaty, Italy. (Paper in press).

[4] M.Cleland, and G.Pageau. Nuclear Instruments and Methods in Physics Research. 1987. B24/25, 967-972.

[5] J.Meissner, M. Abs, M.Cleland, A.Herer, Y. Jongen, F. Kuntz, A.Strasser. Radiation Physics and Chemistry. 2000. Vol. 57, 647-651.

[6] S. Pismenesky, G.Popov, V.Rudychev. Radiation Physics and Chemistry. 2002. Vol. 63, 601-602.

[7] Y.Jongen Abstract book of the $6^{\text {th }}$ Gamma Processing Seminar. 2002. Ottawa. Canada. 